Zbigniew Grande, Institute of Mathematics, Pedagogical University, plac Weyssenhoffa 11, 85-072, Bydgoszcz, Poland

e-mail: grande@wsp.bydgoszcz.pl

\title{
ON SEQUENCES OF MONOTONE FUNCTIONS
}

\begin{abstract}
Several kinds of convergence (including pointwise, monotone, a.c., uniform, ...) in the family of monotone functions are investigated.
\end{abstract}

Let $\mathcal{R}$ denote the set of all reals. Observe that the limit $f$ of a converging sequence of monotone functions $f_{n}: I \mapsto \mathcal{R}$, where $I$ is a nondegenerate interval, is a monotone function. Of course, there is a subsequence $\left(f_{n_{k}}\right)_{k}$, where all functions $f_{n_{k}}$ are decreasing or increasing and consequently, the function $f$ is decreasing or respectively increasing as the limit of the subsequence $\left(f_{n_{k}}\right)_{k}$.

Theorem 1. If $f:[a, b] \mapsto \mathcal{R}$ is an increasing function (i.e. nondecreasing) then there are continuous increasing functions $f_{n}:[a, b] \mapsto \mathcal{R}, n=1,2, \ldots$, such that $f_{n}(a)=f(a), f_{n}(b)=f(b)$ for $n \geq 1$ and $\lim _{n \rightarrow \infty} f_{n}=f$.

Proof. Fix a positive integer $n$ and observe that the set

$$
A=\left\{x \in[a, b] ; \operatorname{osc} f(x) \geq \frac{1}{n}\right\}
$$

is empty or finite. We can assume that $A$ is nonempty. Let

$$
A=\left\{x_{1}, \ldots, x_{k}\right\}, \quad x_{1}<\cdots<x_{k} .
$$

There are closed intervals $I_{i}=\left[a_{i}, b_{i}\right], i \leq k$, such that

$$
\begin{aligned}
& b_{i-1}<a_{i}<x_{i}<b_{i}<a_{i+1} \text { for } i=2, \ldots, k-1 ; \\
& \text { if } a<x_{1} \text { then } a<a_{1}<x_{1}<b_{1} ;
\end{aligned}
$$

Key Words: continuity, monotone functions, convergence, monotone convergence, transfinite convergence, variation

Mathematical Reviews subject classification: Primary: 26A15, 26A21, 26A48

Received by the editors January 18, 1998 


$$
\begin{aligned}
& \text { if } a=x_{1} \text { then } a=a_{1}=x_{1}<b_{1} ; \\
& \text { if } x_{k}<b \text { then } a_{k}<x_{k}<b_{k}<b ; \\
& \text { if } x_{k}=b \text { then } a_{k}<x_{k}=b_{k}=b ; \\
& b_{i}-a_{i}<\frac{1}{n} \text { for } i \leq k ; \\
& f\left(b_{i}\right)-f\left(a_{i}\right)<\operatorname{osc} f\left(x_{i}\right)+\frac{1}{n} \text { for } i \leq k .
\end{aligned}
$$

Since osc $f(x)<\frac{1}{n}$ for each point $x \in[a, b] \backslash A$, there are points $c_{i, j} \in\left(b_{i-1}, a_{i}\right)$, $i=2, \ldots, k, j \leq j(i)$, such that

$$
\begin{gathered}
b_{i-1}=c_{i, 1}<c_{i, 2}<\cdots<c_{i, j(i)-1}<c_{i, j(i)}=a_{i} \text { for } i=2, \ldots, k ; \\
f\left(c_{i, j+1}\right)-f\left(c_{i, j}\right)<\frac{1}{n} \text { for } i=2, \ldots, k .
\end{gathered}
$$

Analogously, if $a<a_{1}\left(b_{k}<b\right)$ there are points $c_{1, j}, j \leq j(1),\left(c_{k+1, j}, j \leq\right.$ $j(k+1)$,) such that

$$
\begin{aligned}
a=c_{1,1}<\cdots<c_{1, j(1)} & =a_{1} \\
\left(b_{k}=c_{k+1,1}<\cdots<c_{k+1, j(k+1)}\right. & =b) \text { for } j \leq j(1) \quad(j \leq j(k+1)) ; \\
f\left(c_{1, j+1}\right)-f\left(c_{1, j}\right) & <\frac{1}{n} \\
\left(f\left(c_{k+1, j+1}\right)-f\left(c_{k+1, j}\right)\right. & \left.<\frac{1}{n}\right) \text { for } j \leq j(1) \quad(j \leq j(k+1)) .
\end{aligned}
$$

Define on the interval $[a, b]$ the following continuous increasing function

$$
f_{n}(x)=\left\{\begin{array}{cll}
f(x) & \text { for } & x \in\left\{a, b, c_{i, j}\right\}, \quad i \leq k+1, \quad j \leq j(i) \\
f(x) & \text { for } & x=x_{i}, \quad i \leq k \\
\text { linear } & \text { otherwise on } & {[a, b] .}
\end{array}\right.
$$

We will prove that $\lim _{n \rightarrow \infty} f_{n}=f$.

If $x \in\left\{a, b, x_{i} ; i \leq k\right\}$ then $f_{n}(x)=f(x)$. Moreover, if $x \in\left[c_{i, j}, c_{i, j+1}\right]$ then $\left|f_{n}(x)-f(x)\right|<\frac{1}{n}$. So, if

$$
x \in[a, b] \wedge \operatorname{dist}(x, A)=\inf \{|u-x| ; u \in A\} \geq \frac{1}{n}
$$

then

$$
\left|f_{n}(x)-f(x)\right|<\frac{1}{n}
$$

For each point $x \in[a, b]$ which is a discontinuity point of the function $f$ there is a positive integer $n$ such that osc $f(x)>\frac{1}{n}$. Consequently, for every $k>n$ we have $f_{k}(x)=f(x)$. 
Now we suppose that $x \in[a, b]$ is a continuity point of the function $f$. Fix a positive real $\eta$ and a positive integer $n$ with $\frac{2}{n}<\eta$. Let

$$
B=\left\{x \in[a, b] \text {; osc } f(x) \geq \frac{1}{n}\right\} \text {. }
$$

Since $x \in[a, b] \backslash B$ and $B$ is a closed set, there is a positive integer $k>n$ with

$$
\left(x-\frac{1}{k}, x+\frac{1}{k}\right) \cap B=\emptyset .
$$

Fix an integer $m>k$. If

$$
E=\left\{y \in[a, b] ; \operatorname{osc} f(y) \geq \frac{1}{m}\right\}
$$

then

$$
\left|f_{m}(x)-f(x)\right|<\frac{1}{m}<\eta
$$

if $\operatorname{dist}(x, E) \geq \frac{1}{m}$ and

$$
\left|f_{m}(x)-f(x)\right|<\operatorname{osc} f(y)+\frac{1}{m}<\frac{1}{n}+\frac{1}{m}<\frac{2}{n}<\eta
$$

for some $y \in E \backslash B$, if $\operatorname{dist}(x, E)<\frac{1}{m}$.

So, $\lim _{n \rightarrow \infty} f_{n}(x)=f(x)$ and the proof is completed.

It is well known that the limit of a decreasing (increasing) sequence of continuous functions is upper (lower) semicontinuous.

Theorem 2. If $f:[a, b] \mapsto \mathcal{R}$ is an upper semicontinuous increasing function then there are continuous increasing functions $f_{n}:[a, b] \mapsto \mathcal{R}, n \geq 1$, such that $f_{n} \geq f_{n+1}>f$ for $n \geq 1$ and $f=\lim _{n \rightarrow \infty} f_{n}$.

In the proof of the above theorem we apply the following sandwich lemma:

Lemma 1. Let $f:[a, b] \mapsto \mathcal{R}$ be an upper semicontinuous increasing (decreasing) function and let $g:[a, b] \mapsto \mathcal{R}$ be a continuous function such that $f(x)<g(x)$ for each $x \in[a, b]$. Then there is a continuous increasing (decreasing) function $h:[a, b] \mapsto \mathcal{R}$ such that $f(x)<h(x)<g(x)$ for all $x \in[a, b]$.

Proof of Lemma 1. We suppose that the function $f$ is increasing. The proof for a decreasing function $f$ is analogous. From the upper semicontinuity of $f$ follows that $f$ is continuous from the right hand. Let

$$
r=\inf \{g(x)-f(x) ; x \in[a, b]\} .
$$


Since the function $g-f$ is positive and lower semicontinuous, the real $r$ is positive. Define the set

$$
A=\left\{x \in[a, b] ; \text { osc } f(x) \geq \frac{r}{5}\right\}
$$

and we observe that it is empty or finite. We can assume that $A$ is nonempty. Let

$$
A=\left\{x_{1}, \ldots, x_{k}\right\}, \quad x_{1}<\cdots<x_{k} .
$$

There are closed intervals $I_{i}=\left[a_{i}, x_{i}\right], i \leq k$, such that

$$
\begin{gathered}
a<a_{i}<x_{i}<a_{i+1}<x_{i+1} \leq b \text { for } i=1, \ldots, k-1 \\
f\left(x_{i}\right)-f\left(a_{i}\right)<\operatorname{osc} f\left(x_{i}\right)+\frac{r}{5} \text { for } i \leq k \\
\left|g(x)-g\left(x_{i}\right)\right|<\frac{r}{5} \text { for } x \in I_{i}, i \leq k .
\end{gathered}
$$

Let $x_{0}=a$. Since osc $f(x)<\frac{r}{5}$ for each point $x \in[a, b] \backslash A$, there are points $c_{i, j} \in\left[x_{i-1}, a_{i}\right], i=1, \ldots, k, j \leq j(i)$, such that

$$
\begin{gathered}
x_{i-1}=c_{i, 1}<c_{i, 2}<\cdots<c_{i, j(i)-1}<c_{i, j(i)}=a_{i} \text { for } i=1, \ldots, k ; \\
f\left(c_{i, j+1}\right)-f\left(c_{i, j}\right)<\frac{r}{5} \text { for } i=1, \ldots, k \text { and } j \leq j(i)-1 .
\end{gathered}
$$

Analogously, if $x_{k}<b$ there are points $c_{k+1, j}, j \leq j(k+1)$, such that

$$
\begin{gathered}
x_{k}=c_{k+1,1}<\cdots<c_{k+1, j(k+1)}=b \\
f\left(c_{k+1, j+1}\right)-f\left(c_{k+1, j}\right)<\frac{r}{5} \text { for } j<j(k+1) .
\end{gathered}
$$

Define on the interval $[a, b]$ a continuous increasing function in the following way:

$$
g_{1}(x)=\left\{\begin{array}{cll}
f(x) & \text { for } & x \in\left\{a, b, c_{i, j}\right\}, \quad i \leq k+1, \quad j \leq j(i) \\
f(x) & \text { for } & x=x_{i}, i \leq k \\
\text { linear } & \text { otherwise on } & {[a, b] .}
\end{array}\right.
$$

If $x \in\left\{a, b, x_{i} ; i \leq k\right\}$ then $g_{1}(x)=f(x)$. Moreover, if $x \in\left[c_{i, j}, c_{i, j+1}\right]$ then $\left|g_{1}(x)-f(x)\right|<\frac{r}{5}$.

Let

$$
h(x)=g_{1}(x)+\frac{r}{4}, \quad x \in[a, b] .
$$

Then $h$ is a continuous increasing function and for

$$
x \in[a, b] \backslash \bigcup_{i \leq k} I_{i}
$$


the inequalities

$$
f(x)<g_{1}(x)+\frac{r}{5}<h(x)<f(x)+r \leq g(x)
$$

are true. If $x \in I_{i}$ for some $i \leq k$ then

$$
\begin{aligned}
f(x) \leq f\left(x_{i}-\right) & =f\left(a_{i}\right)+\frac{r}{5}<g_{1}\left(a_{i}\right)+\frac{r}{4}=h\left(a_{i}\right) \leq h(x) \\
& =f\left(x_{i}\right)+\frac{r}{4} \leq g\left(x_{i}\right)-r+\frac{r}{4}<g\left(x_{i}\right)-\frac{r}{5}<g(x) .
\end{aligned}
$$

So, the function $h$ satisfies to all requirements.

Proof of TheOrem 2. Since the function $f$ is upper semicontinuous, there are continuous functions $g_{n}:[a, b] \mapsto \mathcal{R}$ such that

$$
f(x)<g_{n+1}(x)<g_{n}(x), \quad x \in[a, b], \quad n \geq 1,
$$

and $f=\lim _{n \rightarrow \infty} g_{n}([1])$.

By Lemma 1 there is a continuous increasing function $f_{1}:[a, b] \mapsto \mathcal{R}$ with $f<f_{1}<g_{1}$. Let $h_{2}=\min \left(f_{1}, g_{2}\right)$. By Lemma 2 there is a continuous increasing function $f_{2}:[a, b] \mapsto \mathcal{R}$ with $f<f_{2}<h_{2}=\min \left(f_{1}, g_{2}\right)$. Next by induction, for each positive integer $n>2$ there is a continuous increasing function $f_{n}:[a, b] \mapsto \mathcal{R}$ with $f<f_{n}<\min \left(f_{n-1}, g_{n}\right)$. Consequently, the sequence $\left(f_{n}\right)_{n}$ satisfies all requirements and the proof is completed.

Remark 1. If the function $f$ is upper semicontinuous and increasing (decreasing) then there are continuous increasing (decreasing) functions $g_{n}:[a, b] \mapsto \mathcal{R}$ such that $g_{n}(a)=f(a), g_{n}(b)=f(b), g_{n} \geq g_{n+1}$ for $n \geq 1$ and $\lim _{n \rightarrow \infty} g_{n}=$ $f$.

Without loss of the generality we can suppose that

$$
b=u=\inf \{x \in[a, b] ; f(x)=f(b)\}
$$

and

$$
a=v=\sup \{x \in[a, b] ; f(x)=f(a)\},
$$

since in the contrary case we can consider the reduced function $f /[u, v]$.

We will prove the remark for the case of an increasing function $f$, because the case of a decreasing $f$ is analogous. Let $\left(a_{n}\right)_{n}$ and $\left(b_{n}\right)_{n}$ be sequences such that

$$
a<a_{n+1}<a_{n}<\cdots<a_{1}<b_{1}<\cdots<b_{n}<b_{n+1}<b
$$

and

$$
a=\lim _{n \rightarrow \infty} a_{n}, ; b=\lim _{n \rightarrow \infty} b_{n} .
$$


By Theorem 2, there is a decreasing sequence of continuous increasing functions $f_{n}:[a, b] \mapsto \mathcal{R}$ with $f=\lim _{n \rightarrow \infty} f_{n}$ and $f_{n}>f$ for $n=1,2, \ldots$ Find a strictly increasing sequence $\left(n_{k}\right)_{k}$ of positive integers such that

$$
\begin{gathered}
\lim _{k \rightarrow \infty} n_{k}=\infty ; \\
\frac{f_{n_{k}}\left(a_{k}\right)-f\left(a_{k}\right)}{a_{k}-a}<\min _{i<k} \frac{f_{n_{i}}\left(a_{i}\right)-f\left(a_{i}\right)}{k\left(a_{i}-a\right)} \text { for } k>1 ; \\
\frac{f_{n_{k}}\left(b_{k}\right)-f\left(b_{k}\right)}{b-b_{k}}<\min _{i<k} \frac{f_{n_{i}}\left(b_{i}\right)-f\left(b_{i}\right)}{k\left(b-b_{i}\right)} \text { for } k>1 .
\end{gathered}
$$

For $k \geq 1$ let

$$
\begin{aligned}
& h_{k}(x)=\frac{f_{n_{k}}\left(a_{k}\right)-f\left(a_{k}\right)}{a_{k}-a}(x-a) \text { for } x \in\left[a, a_{k}\right], \\
& h_{k}(x)=\frac{f_{n_{k}}\left(b_{k}\right)-f\left(b_{k}\right)}{b-b_{k}}(b-x) \text { for } x \in\left[b_{k}, b\right]
\end{aligned}
$$

and

$$
g_{k}(x)=\left\{\begin{array}{lll}
f(x)+h_{k}(x) & \text { for } & x \in\left[a, a_{k}\right] \\
f_{n_{k}}(x) & \text { for } & x \in\left[a_{k}, b_{k}\right] \\
f(x)+h_{k}(x) & \text { for } & x \in\left[b_{k}, b\right]
\end{array}\right.
$$

The sequence $\left(g_{k}\right)_{k}$ satisfies all requirements and the proof is completed.

Remark 2. If a function $f:[a, b] \mapsto \mathcal{R}$ is increasing (decreasing) and lower semicontinuous then there is a increasing sequence of continuous increasing (decreasing) functions $f_{n}:[a, b] \mapsto \mathcal{R}$ such that $f_{n}(a)=f(a), f_{n}(b)=f(b)$ for $n \geq 1$ and $\lim _{n \rightarrow \infty} f_{n}=f$.

Proof. It suffices to apply Remark 1 to the function $(-f)$.

We will write that a.c. $\lim _{n \rightarrow \infty} f_{n}=f([2,3])$ if for each point $x$ there is a positive integer $n(x)$ such that for $n>n(x)$ the equality $f_{n}(x)=f(x)$ is true.

Since monotone functions have only countable sets of discontinuity points, we prove the following theorem:

Theorem 3. Suppose that functions $f, f_{n}:[a, b] \mapsto \mathcal{R}$ satisfy the following conditions:

- $f=$ a.c. $\lim _{n \rightarrow \infty} f_{n}$;

- for each integer $n \geq 1$ the set $D\left(f_{n}\right)$ of all discontinuity points of the function $f_{n}$ is countable. 
Then for each nonempty closed set $F \subset[a, b]$ there are an open interval I and a positive integer $k$ such that $I \cap F \neq \emptyset$ and for each point $x \in(F \cap I) \backslash \bigcup_{n} D\left(f_{n}\right)$ and for each integer $n>k$ the equality $f(x)=f_{n}(x)$ is true.

Proof. Since a.c. $\lim _{n \rightarrow \infty} f_{n}=f$, for each point $x \in[a, b]$ there is a positive integer $n(x)$ such that $f(x)=f_{n}(x)$ for all integers $n>n(x)$. For each integer $m \geq 1$ let

$$
A_{m}=\{x \in[a, b] ; n(x)=m\} .
$$

Let $F \subset[a, b]$ be a nonempty closed set. If the set $F$ has an isolated point then the condition of our theorem is satisfied. So, we can assume that $F$ is a perfect set. Since

$$
F=\bigcup_{m}\left(A_{m} \cap F\right),
$$

by the Baire category theorem there is an integer $k \geq 1$ such that the set $A_{k} \cap F$ is of the second category in $F$. Consequently, there is an open interval $I$ such that $I \cap F \neq \emptyset$ and for every open interval $J \subset I$ with $J \cap F \neq \emptyset$ the set $J \cap F \cap A_{k}$ is of the second category in $F$. Since the set

$$
E=\bigcup_{n} D\left(f_{n}\right)
$$

is countable, the set

$$
B=\left(I \cap F \cap A_{k}\right) \backslash E
$$

is dense in $I \cap F$. The restricted functions $f_{n} /([a, b] \backslash E), n \geq 1$, are continuous and for $m, n>k$ and $x \in B$ the equalities

$$
f_{n}(x)=f_{m}(x)=f(x)
$$

are true. So, for $m, n>k$ and for $x \in(I \cap F) \backslash E$ we obtain $f_{m}(x)=f_{n}(x)=$ $f(x)$ and the proof is finished.

Corollary 1. If functions $f_{n}:[a, b] \mapsto \mathcal{R}$ are continuous and increasing (decreasing) and a.c. $\lim _{n \rightarrow \infty} f_{n}=f$ then the function $f$ is increasing (decreasing) and in the class $B_{1}^{*}$ (i.e. for every nonempty closed set $F \subset[a, b]$ there is an open interval $I$ such that $I \cap F \neq \emptyset$ and the restricted function $f /(F \cap I)$ is continuous $[2,3])$.

Proof. This corollary is an evident consequence of the last theorem.

Theorem 4. Suppose that the function $f:[a, b] \mapsto \mathcal{R}$ is increasing (decreasing) and in the class $B_{1}^{*}$. Then there is a sequence of continuous increasing (decreasing) functions $f_{n}:[a, b] \mapsto \mathcal{R}$ with $f=$ a.c. $\lim _{n \rightarrow \infty} f_{n}$. 
Proof. Observe that there are nonempty closed sets $F_{n}, n \geq 1$, such that

$$
\begin{gathered}
{[a, b]=\bigcup_{n} F_{n},} \\
F_{n} \subset F_{n+1}, \quad n \geq 1,
\end{gathered}
$$

and the restricted functions $f / F_{n}$ are continuous ([2]). For each integer $n \geq 1$ the functions $f / F_{n}$ can be extended to a continuous increasing (decreasing) function $f_{n}:[a, b] \mapsto \mathcal{R}$ such that $f_{n}(a)=f(a)$ and $f_{n}(b)=f(b)$. Evidently,

$$
f=\text { a.c. } \lim _{n \rightarrow \infty} f_{n}
$$

and the proof is completed.

Theorem 5. Let $f:[a, b] \mapsto \mathcal{R}$ be a function. The following conditions are equivalent:

(a) $f$ is increasing (decreasing);

(b) There are increasing (decreasing) functions $f_{n}:[a, b] \mapsto \mathcal{R}$ such that $f_{n}(a)=f(a), f_{n}(b)=f(b)$ and the sets $D\left(f_{n}\right)$ of all discontinuity points of $f_{n}, n \geq 1$, are finite and $\lim _{n \rightarrow \infty} V\left(f_{n}-f, a, b\right)=0$, where $V\left(f_{n}-\right.$ $f, a, b)$ denotes the total variation of $f_{n}-f$ on $[a, b]$;

(c) There is a sequence of increasing (decreasing) functions $f_{n}:[a, b] \mapsto \mathcal{R}$ which uniformly converges to $f$ on $[a, b]$ and for which $f_{n}(a)=f(a)$, $f_{n}(b)=f(b)$ and the sets $D\left(f_{n}\right), n \geq 1$, are finite.

Proof. The implication $(c) \Rightarrow(a)$ is evident. Since for each point $x \in[a, b]$ we have

$$
\left|f_{n}(x)-f(x)\right| \leq V\left(f_{n}-f, a, b\right),
$$

we obtain the implication $(b) \Rightarrow(c)$. So, it suffices to prove the implication $(a) \Rightarrow(b)$. Fix an increasing function $f$ and a positive real $\eta$. Observe that the set $D(f)$ is countable. We may assume that $D(f)$ is nonempty. Let

$$
D(f)=\left\{a_{1}, \ldots, a_{k}, \ldots\right\}
$$

Define $g(a)=0$ and for $x \in(a, b]$ let

$$
g(x)=\sum_{a_{i}<x} \operatorname{osc} f\left(a_{i}\right)+(f(x)-f(x-)) .
$$

Put

$$
h(x)=f(x)-g(x) \text { for } x \in[a, b] .
$$


Then the function $h$ is increasing and continuous and $f=h+g$. Since

$$
\sum_{i} \operatorname{osc} f\left(a_{i}\right) \leq f(b)-f(a)<\infty
$$

there is a positive integer $k$ with

$$
\sum_{i>k} \operatorname{osc} f\left(a_{i}\right)<\frac{\eta}{2}
$$

Put $g_{1}(a)=0, g_{1}(b)=g(b)$ and for $x \in(a, b)$ let

$$
g_{1}(x)=\sum_{a_{i}<x ; i \leq k} \operatorname{osc} f\left(a_{i}\right)+(f(x)-f(x-)) .
$$

If

$$
f_{1}(x)=h(x)+g_{1}(x) \text { for } x \in[a, b],
$$

then the function $f_{1}$ is increasing and

$$
f_{1}(a)=f(a), \quad f_{1}(b)=f(b),
$$

the set $D\left(f_{1}\right) \subset\left\{a_{1}, \ldots, a_{k}, b\right\}$ is finite, and

$$
V\left(f_{1}-f, a, b\right)=2 \sum_{i>k} \operatorname{osc} f\left(a_{i}\right)<2 \frac{\eta}{2}=\eta .
$$

This completes the proof for the increasing functions. If $f$ is a decreasing function on $[a, b]$ then we can use the proved part to the function $(-f)$. So, the proof is completed.

Now, denote by $\omega_{1}$ the first uncountable ordinal number and consider a transfinite sequence of monotone functions $f_{\alpha}:[a, b] \mapsto \mathcal{R}, \alpha<\omega_{1}$. We will say that the sequence $\left(f_{\alpha}\right)_{\alpha<\omega_{1}}$ converges to a function $f$ (then we write $\left.\lim _{\alpha} f_{\alpha}=f\right)$ if for each point $x \in[a, b]$ there is a countable ordinal $\alpha(x)$ such that $f(x)=f_{\alpha}(x)$ for $\alpha>\alpha(x)([4])$.

Theorem 6. If a function $f:[a, b] \mapsto \mathcal{R}$ is the limit of a transfinite sequence of monotone functions $f_{\alpha}, \alpha<\omega_{1}$, then there is a countable ordinal $\beta$ such that $f=f_{\alpha}$ for $\alpha>\beta$.

Proof. The assumptions imply the monotonicity of the function $f$. Let $A \subset[a, b]$ be a countable set containing $D(f) \cup\{a, b\}$ which is dense in $[a, b]$. There is a countable ordinal $\beta$ such that

$$
f_{\alpha}(x)=f(x), \quad x \in A, \quad \alpha>\beta .
$$


If $\alpha>\beta$ is a countable ordinal then $f_{\alpha}=f$. Of course, if there is a point $x \in[a, b]$ with $f_{\alpha}(x) \neq f(x)$ then $x \in[a, b] \backslash A$. Consequently, $f$ is continuous at $x$ and there is a positive real $r$ such that $f_{\alpha}(x)$ is not in the interval $(f(x)-$ $r, f(x)+r)$. Since the graph of the restricted function $f / A$ is dense in the graph of $f$, there are points $u, v \in A$ with

$$
f(x)-r<f(u)<f(x)<f(v)<f(x)+r .
$$

But

$$
f_{\alpha}(u)=f(u), \quad f_{\alpha}(v)=f(v)
$$

and $f_{\alpha}$ is monotone, so

$$
f_{\alpha}(x) \in(f(x)-r, f(x)+r),
$$

a contrary. This completes the proof.

Since each nondegenerate interval $I$ is the union of closed intervals $I_{n}$, $n \geq 1$, such that $\operatorname{int}\left(I_{n}\right) \cap \operatorname{int}\left(I_{m}\right)=\emptyset$ for $n \neq m$, we obtain that

Remark 3. Theorems $1,4,5$ and 6 and Remarks 1 and 2 are true for monotone functions $f: I \mapsto \mathcal{R}$ with $f_{n}(a+)=f(a+)$ and $f_{n}(b-)=f(b-)$.

\section{References}

[1] A. M. Bruckner, J. B. Bruckner and B. S. Thomson, Real Analysis, Prentice-Hall, New Jersey, 1994.

[2] A. Császár and M. Laczkovich, Discrete and equal convergence, Studia Sci. Math. Hungar. 10 (1975), 463-472.

[3] R. J. O'Malley, Approximately differentiable functions. The $r$ topology, Pacific J. Math. 72 (1977), 207-222.

[4] W. Sierpiński, Sur les suites transfinies convergentes de fonctions de Baire, Fund. Math. 1 (1920), 132-141. 